\title{
A New Approach to Understanding the Frequency Response of Mineral Oil
}

\author{
Yuan Zhou, Miao Hao, George Chen \\ University of Southampton, UK \\ Yz1d10@ecs.soton.ac.uk
}

\begin{abstract}
Dielectric spectroscopy is non-invasive diagnostic method and can give information about dipole relaxation, electrical conduction and structure of molecules. Since the creation of charge carriers in mineral oil is not only from dissociation but also injection from electrodes, the injection current cannot be simply ignored. The polarization caused by the charge injection has been studied in this paper. Based on our research, if the mobility of the injected charge carriers is fast enough so that they can reach the opposite electrode, the current caused by the injection will contribute only to the imaginary part of the complex permittivity and this part of the complex permittivity will decrease with the frequency with a slope of -1 which is in a good agreement with the experimental result. The classic ionic drift and diffusion model and this injection model will be combined to make an improved model. In this paper, the frequency responses of three different kinds of mineral oils have been measured, and this modified model has been used to simulate the experiment result. Since there is only one unknown parameter in this improved model, a better understanding of the frequency response in mineral oil can be achieved.
\end{abstract} carrier

Keywords-dielectric spectroscopy; injection; fast charge

\section{INTRODUCTION}

Mineral oil, as a highly insulating and non-conducting coolant, is widely used in power industry. It is generally accepted that the quality of the oil can greatly affect the performance of high voltage electrical equipment. The electric polarization in liquid has been studied by Jaffé and a linear approximation has been made [1-3]. Many researchers have studied this issue and several analytical solutions have been developed under various conditions and for different materials [4-10]. Coelho has proposed a model by assuming that the free charges move through the sample and drift towards the electrode and finally result in a macro-dipole [11]. Frood and Gallagher further developed Coelho's theory for liquid sample and reported that Coelho's model was unable to explain the behaviour of the imaginary part of the complex permittivity [12]. Frood and Gallagher thought there might be highly mobile charge carriers in liquid [12].

As shown in literatures, the real part of the complex permittivity of the oil would increase as the frequency goes low, whilst the imaginary part decreases with frequency with a slope approximate to -1 [13-15]. The theoretical analysis of this polarization is generally carried by solving the ionic drift and diffusion equations under the condition that the charge carriers

\author{
Paul Jarman, Gordon Wilson \\ National Grid UK
}

can be totally or partially blocked by the electrode. However, a peak in the imaginary part of the complex permittivity is unavoidable in a frequency ranges from $10^{-3} \mathrm{~Hz}-10^{4} \mathrm{~Hz}$ based on current theories and simulations, which is against the experimental data [1-15]. Therefore, other kinds of charge transportation should be involved. In this paper, this new type of charge carrier is assumed to be the injected charge carriers. The injected charge carriers are charged in the region close to one electrode and get discharged in the vicinity of the other electrode. The charge injection in liquid was first studied by Felici in 1978 [16]. He took account the field distribution in the region that was close to the interface between metal electrode and liquid and the charge transportation mechanisms participating at this interface and an analytic solution has been developed. This ionic injection theory has been widely studied and verified in various kinds of the dielectric liquids under different experimental conditions [17-21].

In our previous work, an ohmic component has been added to the classic ionic drift and diffusion model and this new model can fit the experimental data quite well [22]. In this paper, properties of three types of mineral oils with different aging time will be studied using dielectric spectroscopy method. A new approach based on the electrode polarization and the charge injection will be introduced.

\section{GENERAL THEORY}

A theory of space charge polarization has been developed by Coelho [11]. In his theory, the distribution of charge carriers has been assumed to be linear. With the linearization, the complex permittivity for a liquid sample measured by parallel electrodes system can be denoted as

$$
\begin{gathered}
\varepsilon(\omega)=\varepsilon_{m}\left[\frac{1+i \omega \tau}{i \omega \tau+\tanh \left(k_{\omega} d\right) / k_{\omega} d}\right] \\
\left\{\begin{array}{c}
\tau=\varepsilon_{m} / \sigma \\
k_{\omega}^{2}=\frac{2 n_{0} q^{2}}{\varepsilon_{m} k T}(1+i \omega \tau)
\end{array}\right.
\end{gathered}
$$

where, $\varepsilon_{m}$ is the permittivity of the sample, $\sigma$ is the conductivity, $\omega$ is the angular frequency, $n_{0}$ is the density of charge carriers, $k$ is the Boltzmann constant, $T$ is the absolute temperature, $q$ is the charge that is carried by a mobile charge carrier and $2 d$ is the distance between the two parallel electrodes. 
In our previous work, it has been revealed that when the field is very low, adding an ohmic component to the classical ionic drift and diffusion model can enable both the real part and the imaginary part to be well fitted [22]. Here, the polarization caused by this injection will be studied in depth.

There are two different situations for the migration of the charge injection in liquid. First, the injected charge carriers are unable to reach the opposite electrode in a full cycle. Second, these injected charge carriers can get to the opposite electrode.

To achieve an analytical solution, several assumptions have been made. First, the injecting current density that is in the vicinity of interface between the electrode and the liquid is proportional to the electric field. Second, the field between the two parallel electrodes can be treated as a homogeneous field. Third, the injected charge carriers can be neutralized when they approach the electrode close enough.

In our previous work [23], it has been found out that when these injected charge carriers cannot reach the opposite electrode, the injecting current will only contribute to the imaginary part of the complex permittivity. The polarization caused by the injection under a sine wave field can be denoted as

$$
\left\{\begin{array}{c}
\Delta \varepsilon^{\prime \prime}=\frac{2 q_{i}^{0} \mu_{i}^{2} E_{0}}{\omega^{2} d} \\
\Delta \varepsilon^{\prime}=0
\end{array}\right.
$$

where $\Delta \varepsilon^{\prime \prime}$ and $\Delta \varepsilon^{\prime}$ is the change of the complex permittivity in imaginary part and real part, $E_{0}$ is the magnitude of the sine wave field, $\mu_{i}$ is the mobility of the injected charge carriers and $q_{i}^{0}$ is a constant change density that related to the nature of the electrode and the dielectric liquid [23].

If the injected charge carriers are fast enough to reach the opposite electrode, the polarization cause by injection can be denoted as

$$
\left\{\begin{array}{c}
\Delta \varepsilon^{\prime \prime}=\frac{q_{i}^{0} \mu_{i}}{\omega}=\frac{\sigma_{i}}{\omega} \\
\Delta \varepsilon^{\prime}=0
\end{array}\right.
$$

where $\sigma_{i}$ is the conductivity contributed from the charge injection. As observed from the experimental result, the imaginary part of the complex permittivity always decreases with the frequency in a slope of approximate -1. Therefore, the mobility of these injected charge carriers should be quite fast so that they are able to travel to the opposite electrode [23].

On considering the polarization caused by the injection, Coelho's space charge polarization theory should be modified as

$$
\varepsilon(\omega)=\varepsilon_{m}\left[\frac{1+i \omega \tau^{\prime}}{i \omega \tau^{\prime}+\tanh \left(k_{\omega}^{\prime} d\right) / k_{\omega}^{\prime} d}\right]-i \frac{\sigma_{i}}{\omega}
$$

with

$$
\left\{\begin{array}{c}
\tau^{\prime}=\varepsilon_{m} /\left(\sigma-\sigma_{i}\right) \\
k_{\omega}^{\prime 2}=\frac{2 n_{0}\left(\sigma-\sigma_{i}\right) q^{2}}{\sigma \varepsilon_{m} k T}\left(1+i \omega \tau^{\prime}\right)
\end{array}\right.
$$

III. COMPARATION BETWEEN THE MODIFIED SPACE CHARGE POLARIZATION MODEL AND THE EXPERIMENTAL RESULT

Here, the frequency responses of three different types of mineral oils, oil A (fresh oil), oil B (aged 10 years) and oil C (aged 50 years), will be measured. Although there are differences in these oils when they were new, their dielectric characteristics are likely to have been sufficiently similar. Thus, the changes observed experimentally are most likely owing to the effect of age rather than any differences between the original oil. The detail of these measurements can be found in our previous work [22].

In order to study the injection in these different kinds of oils, the ratio of conductivity contributed by the injection over the total conductivity, $\alpha$, which has been defined as

$$
\alpha=\frac{\sigma_{i}}{\sigma}
$$

will be investigated in this paper [22].

Numerical simulations are carried out with only one unknown parameter $\alpha$. Conductivity will be calculated directly from the imaginary part of the complex permittivity. Permittivity is obtained from the real part of the complex permittivity at $1 \mathrm{~Hz}$ experimentally. The mobility of these charge carriers is assumed to be proportional to the viscosity of the oil. The viscosities of these mineral oils can be found in our previous work [22].

The experimental results of the real part of the complex permittivity of these three kinds of mineral oils are illustrated in Figs. 1-3. The real parts of the relative complex permittivity of the all three kinds of mineral oil are around 2.1-2.4 in frequency ranges from $1 \mathrm{~Hz}$ to $100 \mathrm{~Hz}$. The frequency response of all three kinds of mineral oils share similar patterns. The real part of complex permittivity of mineral oil does not change much when the frequency is above $1 \mathrm{~Hz}$, and then increases as the frequency decreases. The real part of the complex permittivity of the oil $\mathrm{B}$ and oil $\mathrm{C}$ increase faster than that of the oil A. Because when the mineral oil is aged, extra charge carriers are generated and the conductivity of the oil increases. Since there are more charge carriers, the space charge polarization should become more significant and a high real part of the complex permittivity can be observed. When the temperature goes higher, the curves of the real part of the complex permittivity shift towards the high frequency region. At high temperature, there are more charge carriers in the oil and these charge carriers move faster. The curves in Figs 1-3 are calculated using Eq. (5). It seems this modified space charge polarization theory can fit the frequency response of all three kinds of mineral oil quite well. The complex permittivity that acquired from the simulation are also constant at high frequency, whilst it shows an increase as the frequency decreases. The space charge polarizations that calculated from Eq. (5) and (6) become more significant when the oil is aged. 
Thus, the calculated value is in a good agreement with the experimental result.

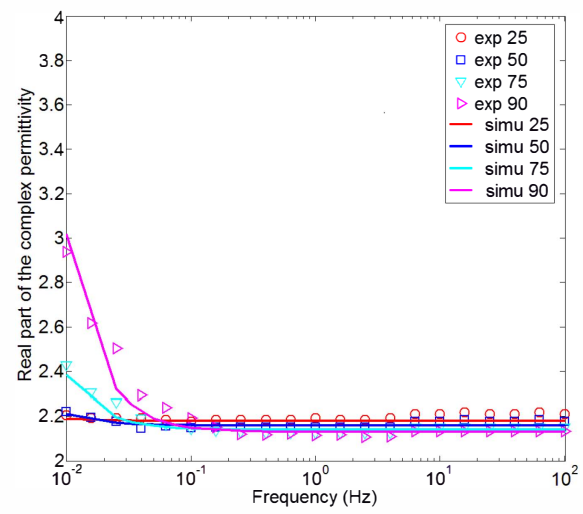

Fig. 1. Theoretical and experimental results of the real part of the complex permittivity of oil A.

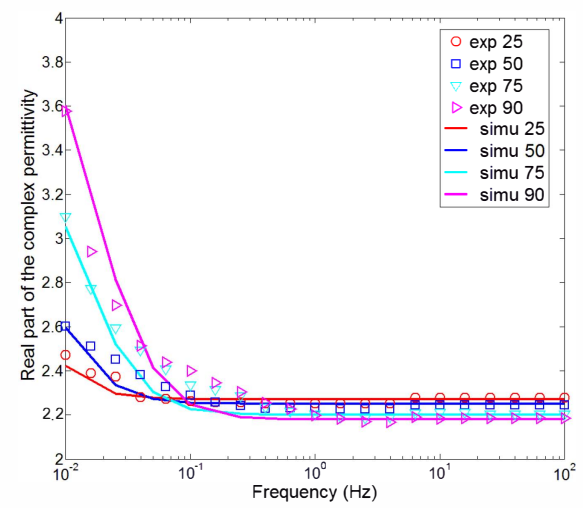

Fig. 2. Theoretical and experimental results of the real part of the complex permittivity of oil B.

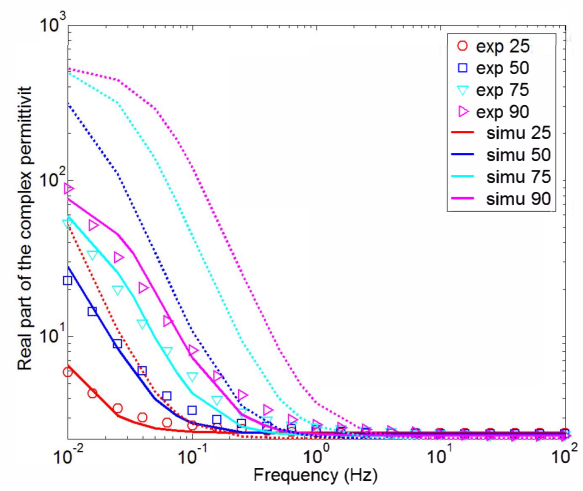

Fig. 3. Theoretical and experimental results of the real part of the complex permittivity of oil C. The dotted line are calculated value using Eq. (1)

The theoretical and the experimental results of the imaginary part of the complex permittivity of these three kinds of mineral oils are illustrated in Figs. 4-6. The imaginary part of the complex permittivity of the mineral oils decreases with the frequency with a slope of approximately -1. It indicates that the conductivity plays an important role in the frequency response of mineral oil in a frequency range of $0.01 \mathrm{~Hz}-100 \mathrm{~Hz}$ [24]. Oil A has the lowest conductivity, oil B has a medium conductivity and oil $\mathrm{C}$ has the highest conductivity. Not surprisingly, the conductivity of mineral oil increases with aging as more charge carriers will be generated due to physical and chemical degradation [25]. The curves of the imaginary part of the complex permittivity shift towards the higher frequency region when the temperature becomes high. The ionic dissociation rate increases with the temperature whilst the viscosity of mineral oil decreases with the temperature. When the temperature rises, there will be more charge carriers and the mobility of these charge carriers will be higher. Thus, a higher temperature can result in a higher conductivity. This modified polarization model can fit the imaginary part of the complex permittivity of all three kinds of mineral oil well at four different temperatures.

The curves of the frequency dependent complex permittivity of oil $\mathrm{C}$ that calculated from Eq. (1) are shown in Fig. 3 and 6 . The values of the real part of the complex permittivity that calculated from Eq. (1) are higher than the values that obtained experimentally when the frequency is below $1 \mathrm{~Hz}$. The curves for the imaginary part of the complex permittivity that calculated from Eq. (1) reach a peak as the frequency decreases. Therefore, Coelho's space charge polarization theory is still not a perfect theory and it needs to be improved.

In all, both the real part and imaginary part of the complex permittivity of the mineral oil calculated from the modified polarization model have a good agreement with the results obtained from the experiments. It seems if part of the charge carriers are injected from the electrode, both the real and imaginary part of the complex permittivity can be fitted.

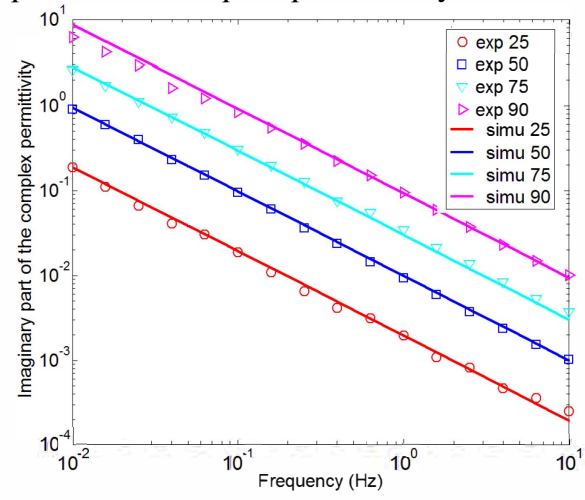

Fig. 4. Theoretical and experimental results of the imaginary part of the complex permittivity of oil A.

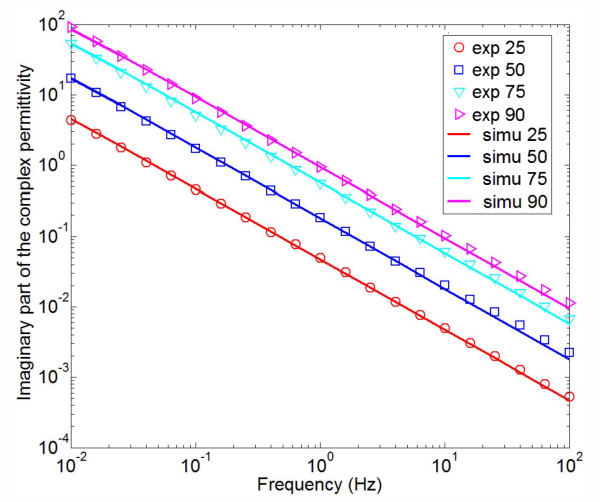

Fig. 5. Theoretical and experimental results of the imaginary part of the complex permittivity of oil $\mathrm{B}$. 


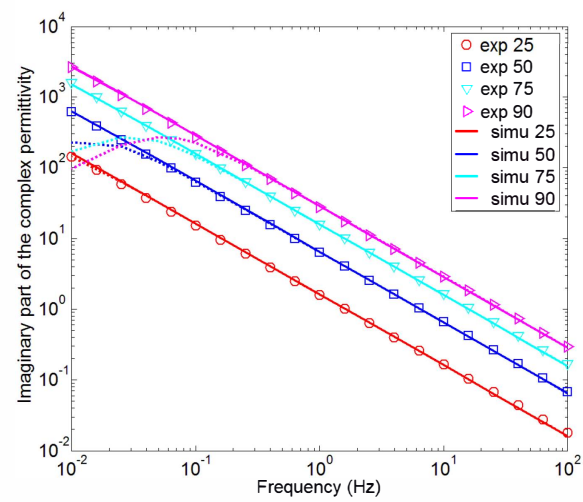

Fig. 6. Theoretical and experimental results of the imaginary part of the complex permittivity of oil $\mathrm{C}$. The dotted line are calculated value using Eq. (1)

The coefficient 1- $\alpha$ of three different types of mineral oils are shown in Table I. When the oil is aged, the injected charge carriers will be the main charge carriers in the mineral oil. Also, a higher temperature can result in a smaller proportion of the first kind of the charge carriers and a larger amount of the second kind of charge carriers. Therefore, the injection can be enhanced by aging and high temperature.

TABLE I. TEMPERAUTE DEPENDENCE OF $1-\alpha$

\begin{tabular}{|c|c|c|c|c|}
\hline $1-\alpha$ & $25^{\circ} \mathbf{C}$ & $50^{\circ} \mathbf{C}$ & $75^{\circ} \mathbf{C}$ & $90^{\circ} \mathbf{C}$ \\
\hline Oil A & 0.90 & 0.70 & 0.60 & 0.45 \\
\hline Oil B & 0.40 & 0.15 & 0.10 & 0.065 \\
\hline Oil C & 0.14 & 0.11 & 0.08 & 0.07 \\
\hline
\end{tabular}

The above injection induced polarization theory is developed based on the assumption that the internal field distortion is not serious. When this frequency-domain measurement is carried out under high electric field, more charge carriers will be created and the field distortion can be significant. Thus, this modified model might not be valid if the field is high.

\section{CONCLUSION}

The present work is concentrated on the analysis of dispersion of dielectric permittivity of mineral oil. The conductivity of mineral oil will increase with aging and temperature. The real part of the complex permittivity increases faster at a low frequency when the oil is aged, whilst the imaginary part of permittivity decrease with the frequency with a slope of -1 regardless of the conductivity.

The modified model can fit the experimental data well and the ratio of the conductivity contributed by injection over the total conductivity might be used in the liquid insulation diagnostics in power industry.

\section{ACKNOWLEDGMENT}

The authors are grateful to the National Grid for their financial support.

\section{REFERENCES}

[1] G Jaffé, "Theory of Conductivity of Semiconductors", Phys. Rev, Vol. $85,354-363,1952$
[2] H. C. Chang and G. Jaffé, "Polarization in Electrolytic Solutions. Part I. Theory", J. Chem. Phys. Vol. 20, 1071-1087, 1952

[3] G. Jaffé and C. Z. LeMay, "On Polarization in Liquid Dielectrics", J. Chem. Phys. Vol. 21, 920-928, 1953

[4] J. R. Macdonald, "Theory of ac Space-Charge Polarization Effects in Photoconductors, Semiconductors, and Electrolytes" Phys. Rev. Vol. 92, 4-17 1953

[5] R. J. Friauf, "Polarization Effects in the Ionic Conductivity of Silver Bromide", J. Chem. Phys. Vol. 22, 1329-1338, 1954.

[6] F. Stern and C. Weaver "Dispersion of dielectric permittivity due to space-charge polarization", J. Phys. C: Solid State Phys. Vol. 3, 17361746,1970

[7] M. Iwamoto, "The dielectric dispersion of insulating films with long-range movements of charge carriers" J. Appl. Phys. Vol. 77, 53145321,1995

[8] R. J. Klein, S. Zhang, S. Dou, B. H. Jones, R. H. Colby, and J. Runt, "Modeling electrode polarization in dielectric spectroscopy: Ion mobility and mobile ion concentration of single-ion polymer electrolytes", J. Chem. Phys. Vol. 124, 1449032006

[9] A. Sawada, "Dielectric process of space-charge polarization for an electrolytic cell with blocking electrodes", J. Chem. Phys. Vol. 129, 0647012008

[10] A. Sawada "Space-charge polarization of a dilute electrolytic cell in the presence of diffuse double layers", J. Appl. Phys. Vol. 112, 0441042012

[11] R. Coelho, Physics of Dielectrics for the Engineer Elsevier Pub. Co, New York 1979

[12] D. G. Frood and T. J. Gallagher, "Space-charge dielectric properties of water and aqueous electrolytes", J. Mol. Liq. Vol. 69, 183-200, 1996.

[13] W. S. Zaengl, "Dielectric spectroscopy in time and frequency domain for HV power equipment. I. Theoretical considerations", IEEE Electrical Insulation Magazine, Vol. 19, 5-19, 2003

[14] C. T. Dervos, C. D. Paraskevas, P. D. Skafidas, and N. Stefanou, "Dielectric spectroscopy and gas chromatography methods applied on high-voltage transformer oils" IEEE Trans. Dielectr. Electr. Insul., Vol.13, 586-592, 2006

[15] A. A. Shayegani Akmal, H. Borsi and E. Gockenbach, "Dielectric behavior of insulating liquids at very low frequency", IEEE Trans. Dielectr. Electr. Insul., Vol.13, 532-538 2006

[16] N.Felici J.P.Gosse "Injection d'ions par des électrodes métalliques dans les hydrocarbures liquides de résistivité élevée" Rever. Phys. Appl., 14, 1979

[17] A. Alj, A. Denat, J. P. Gosse and B. Gosse "Creation of Charge Carriers in Nonpolar Liquids", IEEE Trans. Electr. Insul. Vol.20, 221-231 1985

[18] A. Denat, B. Gosse and J. P. Gosse "Ion injection in hydrocarbons", J. Electrostatics, vol. 7, pp.205 -225 1979 A. Denat, B. Gosse, J.P. Gosse, J. Electrostatics. 11179 (1982)

[19] A. Denat, B. Gosse and J. P. Gosse "High field dc and ac conductivity of electrolyte solutions in hydrocarbons", J. Electrostatics, vol. 11, pp.179-194 1982

[20] M. Nemamcha, J. P. Gosse, A. Denat and B. Gosse "Temperature Dependence of Ion Injection by Metallic Electrodes into Non-Polar Dielectric Liquids", IEEE Trans. Electr. Insul. Vol.22 459-465 1987

[21] F.Pontiga A. Castellanos "The Effect of Field-Enhanced Injection and Dissociation on The Conduction of Highly Insulating Liquids" IEEE Transactions on Dielectrics and Electrical Insulation Vol3, 792-799, 1996

[22] Y Zhou, M Hao, G Chen, G Wilson and P Jarman "Space charge Polarization in Insulating Mineral Oil" Proc. IEEE CEIDP, Shenzhen, China, Oct, 2013, vol 1, p.587

[23] Y Zhou, M Hao, G Chen, G Wilson and P Jarman "Study of the dielectric response in mineral oil using frequency-domain measurement" J. Appl. Phys. Vol. 115, 124105, 2014

[24] F. Kremer, A. Schonhals Broadband Dielectric Spectroscopy Springer 2003

[25] R. Bartnikas Engineering Dielectrics Vol. III, Electrrical Insulating Liquids, ASTM, 1994 